\title{
Robust Image Content Authentication Using Perceptual Hashing and Watermarking
}

\author{
Li Weng ${ }^{1}$, Rony Darazi ${ }^{2}$, Bart Preneel $^{1}$, Benoît Macq $^{2}$, and Ann Dooms ${ }^{3}$ * \\ 1 Katholieke Universiteit Leuven \\ 2 Université catholique de Louvain \\ 3 Vrije Universiteit Brussel
}

\begin{abstract}
Perceptual hashing is a promising tool for multimedia content authentication. Digital watermarking is a convenient way of data hiding. By combining the two, we get a more efficient and versatile solution. In a typical scenario, multimedia data is sent from a server to a client. The corresponding hash value is embedded in the data. The data might undergo incidental distortion and malicious modification. In order to verify the authenticity of the received content, the client can compute a hash value from the received data, and compare it with the hash value extracted from the data. The advantage is that no extra communication is required - the original hash value is always available and synchronized. However, on the other hand, image quality can be degraded due to watermark embedding. There is interesting interaction between hashing and watermarking. We investigate this issue by proposing a content authentication system. The hash algorithm and the watermarking algorithm are designed to have minimal interference. This is achieved by doing hashing and watermarking in different wavelet subbands. Through extensive experiments we show that the parameters of the watermarking algorithm have significant influence to the authentication performance. This work gives useful insights into the research and practice in this field.
\end{abstract}

\section{Introduction}

Multimedia is an important element of digital life. Recent technology development has made it easy to produce and distribute multimedia content. While

\footnotetext{
* This work was supported in part by the Research Council K.U.Leuven: GOA TENSE (GOA/11/007), by the IAP programme P6/26 BCRYPT of the Belgian State (Belgian Science Policy), by the European Commission through the ICT programme under contract ICT-2007-216676 ECRYPT II, and by the IBBT/AQUA project with the involved companies. IBBT (Interdisciplinary Institute for BroadBand Technology, Gaston Crommenlaan 8, Ghent) is a research institute founded in 2004 by the Flemish Government and the involved institutions. Additional support was provided by the FWO (Fonds Wetenschappelijk Onderzoek) within the project G.0206.08 "Perceptual Hashing and Semi-fragile Watermarking" and G.0213.11N "Watermarking, Encryption and Transcoding", and by the Post-Doctoral Fellowship of Peter Schelkens. The original publication is available at www.springerlink.com.
} 
there is abundant media content distributed in a large scale system, an important issue is trustworthiness protection. Since software for editing digital content is easily accessible, modified or synthesized content often appears. Unless one knows the original content, it is difficult to find out whether the content has been professionally modified.

In this work, we focus on the trustworthiness protection of images and video. In a heterogeneous network, there are servers, clients, and intermediate nodes with different computing capabilities. Clients receive multimedia data from servers through intermediate nodes that form a distribution chain. The distribution chain is not perfectly reliable, due to the following issues:

- Incidental distortion - the content may undergo re-encoding, e.g. a format change or re-compression, since it is necessary to adjust the data stream according to the client's capability and the network condition. Properties, such as resolution, contrast, etc., may change.

- Malicious modification - there might be malicious nodes that modify or replace the content.

In such a circumstance, an important question of the client is whether the received content is authentic.

The above problem is easy when the original content is available for comparison, but in practice it is usually not the case. When the original content is not available, a possible solution is to generate a hash value on the server side and send it securely to the client side. The hash value is a compact abstract of the content. A client can re-generate a hash value from the received content, and compare it with the original hash value. If they match, the content is considered as authentic. Conventionally, there exist cryptographic hash algorithms for data authentication, such as MD5, SHA-1 [1]. However, they are not suitable for multimedia data, because they are extremely sensitive and do not tolerate any change of the data. In order to correctly authenticate the content even when it has undergone some incidental distortion, the hash value must possess some robustness. Therefore, a new generation of hash algorithms has emerged in the multimedia domain, called robust or perceptual hash (PH) algorithms [2].

Perceptual hash algorithms enable robust content authentication, whereas sometimes it is not easy to setup a secure channel for transmitting hash values. Therefore, a more convenient approach comes fourth - instead of sending through a secure channel, we can deliver the hash value by imperceptibly embedding it into the content itself using robust digital watermarking techniques. The new approach avoids extra communication, and ensures that the original hash value is always available and synchronized. The authentication procedure is simple: a client extracts the original hash value from the watermarked data, and compares it with the re-computed hash value. Such a system was envisioned by Fridrich and Goljan in $[3,4]$. They motivated the usefulness of content-dependent watermarks and designed a robust hash algorithm. However, so far there is no practical design and in-depth study on a complete content authentication system based on perceptual hashing and robust watermarking. 


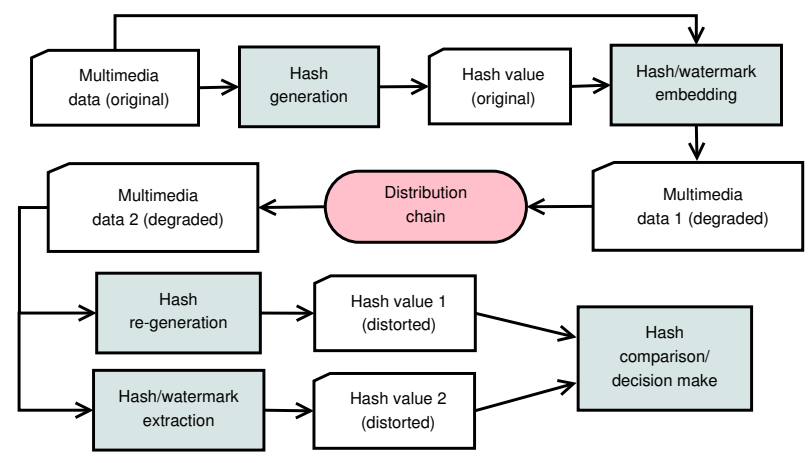

Fig. 1. Diagram of image content authentication.

In this work, we propose a novel content authentication system by combining perceptual hashing and robust watermarking. We use the hash algorithm to essentially achieve content authentication, and focus on the authentication performance under the influence of watermarking. We define the overall system performance as the authentication performance and the image quality loss by watermarking. The proposed system is extensively tested and exhibit good performance. The results justify the validity of the design and serve as a reference for research and practice in this field.

\section{System requirement and overview}

The content authentication system includes an image hash algorithm and a watermarking algorithm. They have their own requirements, but also interact. The schematic diagram of content authentication is shown in Fig. 1.

\subsection{The image hash system}

A perceptual hash value must fairly represent the corresponding content. The hash algorithm should possess the following properties:

- Compactness - a hash value is compact;

- Robustness - hash computation is insensitive to a certain level of distortion to the input image;

- Discriminability - perceptually different content results in significantly different hash values;

- Key dependence - hash generation depends on a key.

We define perceptually similar images as images generated by certain incidental distortion. If two images are not perceptually similar, they are considered as perceptually different. In the content authentication scenario, the distance $d$ between two hash values is compared with a threshold $T$. The decision is made from two hypotheses: 1) $\mathbb{H}_{0}$ - the images correspond to different content; 2) $\mathbb{H}_{1}$ 
- the images correspond to similar content. If $d \leq T$, we choose $\mathbb{H}_{1}$; otherwise we choose $\mathbb{H}_{0}$.

The overall performance of the hash algorithm can be characterized by the true positive rate $P_{d}$ and the false positive rate $P_{f}$. A good algorithm should suppress the false positive rate while maintaining a high true positive rate. The relationship between $P_{d}$ and $P_{f}$ is known as the receiver operating characteristic (ROC) curve. In order to choose the most suitable threshold value, we also need to take into account of the false negative rate $P_{m}$. By definition, $P_{m}=1-P_{d}$. Actually $P_{f}$ and $P_{m}$ are contradicting requirements. Different applications give their own bias towards $P_{f}$ or $P_{m}$. By default, we choose the equal error rate (EER) point, where $P_{f}=P_{m}$, as the working point.

\subsection{The watermarking system}

The watermarking scheme, as a method for data hiding, generally concerns the following issues:

- Payload capacity - the scheme can carry a certain amount of information;

- Robustness - the scheme should withstand certain distortion to the image;

- Perceptual quality - the watermark has little impact on the image quality;

- Key dependence - the algorithm depends on a key.

The performance of a watermarking scheme depends on the embedding strength and the size of the payload.

In this work, we use the quantization index modulation (QIM) [5]. It is very robust to noise. In addition to the high capacity-robustness trade-offs, QIM methods are simple and have a small computational cost. Watermark detection in QIM is performed without access to the original data or the original watermark.

\subsection{Performance evaluation}

The overall performance is a balance between the authentication performance under incidental distortion and the quality loss due to watermarking. Typically, the watermark embedding also brings distortion to the original image, so it might affect the re-computation of the hash value. This interference, denoted as $I_{w h}$, must be small.

Besides, the parameters of the two algorithms also influence each other's performance. For example, increasing the hash size typically increases the discrimination performance, but also increases the payload and decreases the robustness of the watermark. Increasing the watermark embedding strength may increase $I_{w h}$, but on the other hand achieves better robustness of the watermark.

Therefore, the design of such a system involves the interaction between the two components. We need to optimize the authentication performance and the perceptual quality. In practice, we can fix one of them and try to get the best performance for the other. 


\section{The proposed scheme}

In the proposed scheme, the adopted watermarking algorithm is the spread transform dither modulation (STDM), a variant of QIM. It exhibits robustness to requantization and meanwhile maintains low complexity. Instead of quantizing the host signal $x$ itself, the quantization occurs entirely in the projection of the host signal $x$ onto a randomly generated vector $p$, which depends on a secret key $K_{w}$. Consequently, the embedding of each information bit occurs in the projection of the host signal $x$ by quantizing it with a uniform, scalar, dithered quantizer [5]. In this method, each bit of the watermark message is spread into $n$ samples instead of one sample.

Consider a host signal $\mathbf{x}=\left[x_{1}, x_{2}, \ldots, x_{N}\right]^{T}$ of length $N$. We break the whole signal into segments with equal length $n$, and embed one bit into each segment $\mathbf{x}_{\mathbf{i}}(i=1,2, \cdots,\lfloor N / n\rfloor)$. This bit is spread in the projection of the segment onto a random vector $\mathbf{p}$. The watermarked signal is:

$$
\mathbf{y}_{\mathbf{i}}=\mathbf{x}_{\mathbf{i}}+\left(Q_{m}\left(\mathbf{x}_{\mathbf{i}}^{T} \mathbf{p}\right)-\mathbf{x}_{\mathbf{i}}^{T} \mathbf{p}\right) \mathbf{p}, \quad m \in\{0,1\},
$$

where $Q_{m}$ is a quantizer with quantization step $\Delta$. The STDM decoder makes a decision based on the projection of the channel output $\mathbf{y}_{\mathbf{i}}$ onto the spreading vector $\mathbf{p}$. The detection can be performed with a minimum distance decoder:

$$
\hat{m}=\arg \min _{m \in\{0,1\}}\left|\mathbf{y}_{\mathbf{i}}^{T} \mathbf{p}-Q_{m}\left(\mathbf{y}_{\mathbf{i}}^{T} \mathbf{p}\right)\right| .
$$

The watermarking space is the two-dimensional discrete wavelet transform space. We use the biorthogonal 4.4 wavelet. The embedding happens on the third decomposition level of luminance component using the horizontal and vertical ( $\mathrm{LH}$ and HL) subbands.

Regarding perceptual hashing, we need a hash algorithm which is representative, light-weight, and has moderate performance. Additionally, it is easily configurable and compatible with the watermarking algorithm. Based on these considerations, we design an image hash algorithm which also works in the wavelet domain. The advantage of using the same domain as the watermarking algorithm is that the influence of watermark embedding to the hash re-computation $I_{w h}$ can be minimized by doing watermarking and hashing in different wavelet subbands. The algorithm generates an $L_{h}$-bit $\mathrm{PH}$ value which depends on a secret key $K_{h}$. It has the following steps:

1. Convert the input image to gray-scale and proportionally resize it to make the maximum dimension equal to 512 pixels;

2. Compute $X$ so that level $X$ has more than $L_{h}$ wavelet coefficients;

3. Two-dimensional wavelet transform until level $X$;

4. Apply a two-dimensional discrete cosine transform (DCT) to the approximate (LL) subband;

5. Extract the sign bits of the first $L_{h}$ DCT coefficients using a zigzag scan, excluding the DC; 
6. Concatenate all the extracted bits and perform a random permutation according to $K_{h}$.

When two hash values are compared, the bit error rate (BER), i.e., the normalized Hamming distance, is used as the distance metric.

Since the hash generation happens in the approximation subband and the watermark embedding happens in the detail subbands, hash re-computation is almost not affected by watermark insertion.

\section{Experiment results}

The system performance depends on the parameters of both algorithms. In this work, we investigate how the watermarking algorithm affects the content authentication performance. We fix the hash size to 128 bits, and vary the watermarking parameters $n$ and $\Delta$, i.e., the length of the spreading vector and the quantization step. The following values are used:

- n-16, 32, 64, 128, 256;

- $\Delta-40,60,80,100,120,160,200,240$.

For each combination of $n$ and $\Delta$, we evaluate the content authentication performance by three tests:

- Robustness test - hash values of original images and their distorted versions are compared;

- Discrimination test - hash values of different images are compared;

- Hypothesis test - the false positive rate and and false negative rate are computed for all thresholds.

A database of 100 images is used. They are natural scene photos of different genres such as architecture, art, humanoids, landscape, objects, vehicles, etc. In the robustness test, distorted versions are generated for each original image. Five kinds of incidental distortion are simulated. Additionally, we also simulate malicious modification by block tampering - an image block is replaced with a different block randomly chosen in the same image. The size of a block is $1 / 64$ of the image area. The distortions are listed in Table 1. Ideally, our system should be able to distinguish incidental and malicious distortion.

There are 100 original images and 4200 distorted images. Hash values are generated for all of them. Note that a hash value of an original image is generated by the following procedure: 1)embedding the original hash value into the image; 2) distort the image; 3 ) extract the watermark. The hypothesis test is done by pair-wise comparing all the hash values (excluding those by block tampering since they are not considered as authentic). There are 4300 runs of hash generation and $\left(\begin{array}{c}3600 \\ 2\end{array}\right)=6,478,200$ hash comparisons for each $\{n, \Delta\}$ pair.

The content authentication performance is represented by the EER, i.e., $P_{f}$ or $P_{m}$ when they are equal. Different EER values w.r.t different $\{n, \Delta\}$ combinations are listed in Table 2. A lower value means better performance. The 
Table 1. List of legitimate and malicious distortion.

\begin{tabular}{l|l}
\hline Distortion & Parameter range (step) \\
\hline AWGN & PSNR 20-50 dB (5) \\
JPEG compression & Quality factor (QF) 10-70 (10) \\
Gaussian filtering & Window size 7 - 19 (2) \\
Sharpening & Strength 0.1 $-0.7(0.1)$ \\
Gamma correction & Gamma 0.5 $-1.7(0.2)$ \\
\hline Block tampering & No. of blocks 1-7 (1) \\
\hline
\end{tabular}

Table 2. EER values for different $\{n, \Delta\}$ combinations.

\begin{tabular}{l|l|l|l|l|l|l|l|l}
\hline$n \backslash \Delta$ & 40 & 60 & 80 & 100 & 120 & 160 & 200 & 240 \\
\hline 16 & .0633 & .0244 & .0067 & .0061 & .0044 & .0021 & .0019 & .0010 \\
32 & .0888 & .0494 & .0113 & .0065 & .0061 & .0041 & .0028 & .0020 \\
64 & .1312 & .0804 & .0508 & .0181 & .0092 & .0044 & .0032 & .0022 \\
128 & .2248 & .1066 & .0639 & .0507 & .0480 & .0123 & .0047 & .0046 \\
256 & .3656 & .2327 & .1303 & .0809 & .0613 & .0508 & .0264 & .0174 \\
\hline
\end{tabular}

image quality loss can be measured by comparing the images before and after watermark embedding. Three quality metrics are considered here: the peak signal-to-noise ratio (PSNR), the signal-to-noise ratio (SNR), and the structural similarity (SSIM) [6]. The average results are listed in Table 3, 4, and 5 respectively. A higher value means better perceptual quality. Based on the above

Table 3. Average PSNR (dB) values for different $\{n, \Delta\}$ combinations.

\begin{tabular}{l|l|l|l|l|l|l|l|l}
\hline$n \backslash \Delta$ & 40 & 60 & 80 & 100 & 120 & 160 & 200 & 240 \\
\hline 16 & 53.10 & 49.94 & 47.68 & 45.90 & 44.43 & 42.10 & 40.28 & 38.78 \\
32 & 56.28 & 52.79 & 50.62 & 48.92 & 47.50 & 45.23 & 43.44 & 41.97 \\
64 & 60.89 & 56.07 & 53.55 & 51.82 & 50.45 & 48.25 & 46.53 & 45.10 \\
128 & 69.00 & 61.16 & 57.62 & 55.47 & 53.95 & 51.71 & 50.03 & 48.64 \\
256 & 84.71 & 70.26 & 64.46 & 61.23 & 59.11 & 56.36 & 54.51 & 53.11 \\
\hline
\end{tabular}

results, there are two observations:

1. The authentication performance increases with smaller $n$ and larger $\Delta$;

2. The image quality loss increases with smaller $n$ and larger $\Delta$.

Implicitly, there is a third relationship on watermarking:

3. The security of watermarking decreases with smaller $n$ and larger $\Delta$.

This is because a longer spreading vector is in general more difficult to discover; on the other hand, when the quantization is stronger, the watermark pattern becomes more noticeable to an attacker.

In practice, a user can first choose an acceptable image quality level and a watermarking security level, then he can find the corresponding authentication 
Table 4. Average SNR (dB) values for different $\{n, \Delta\}$ combinations.

\begin{tabular}{l|l|l|l|l|l|l|l|l}
\hline$n \backslash \Delta$ & 40 & 60 & 80 & 100 & 120 & 160 & 200 & 240 \\
\hline 16 & 46.34 & 43.18 & 40.92 & 39.14 & 37.67 & 35.34 & 33.51 & 32.02 \\
32 & 49.51 & 46.03 & 43.86 & 42.15 & 40.74 & 38.47 & 36.68 & 35.21 \\
64 & 54.13 & 49.31 & 46.79 & 45.06 & 43.68 & 41.49 & 39.77 & 38.34 \\
128 & 62.24 & 54.40 & 50.86 & 48.71 & 47.19 & 44.95 & 43.27 & 41.88 \\
256 & 77.95 & 63.50 & 57.70 & 54.47 & 52.35 & 49.60 & 47.75 & 46.35 \\
\hline
\end{tabular}

Table 5. Average SSIM values for different $\{n, \Delta\}$ combinations.

\begin{tabular}{l|l|l|l|l|l|l|l|l}
\hline$n \backslash \Delta$ & 40 & 60 & 80 & 100 & 120 & 160 & 200 & 240 \\
\hline 16 & .9976 & .9953 & .9923 & .9888 & .9847 & .9750 & .9635 & .9507 \\
32 & .9988 & .9974 & .9959 & .9941 & .9920 & .9869 & .9808 & .9739 \\
64 & .9996 & .9988 & .9978 & .9968 & .9958 & .9932 & .9901 & .9866 \\
128 & .9999 & .9996 & .9991 & .9986 & .9980 & .9968 & .9954 & .9937 \\
256 & 1.000 & .9999 & .9998 & .9996 & .9994 & .9988 & .9982 & .9976 \\
\hline
\end{tabular}

performance, or vice versa. For example, we can choose $n=64, \Delta=120$ to get a PSNR around $50 \mathrm{~dB}$ in Table 3 . The corresponding EER is $P_{f}=P_{m}=0.0092$ in Table 2. The detailed authentication performance results are presented below.

In order to evaluate the robustness of watermark embedding, we compare the original hash value with the extracted one after distortion. The average results are listed in Table 6 . One can see that in most cases the original hash value has a tiny difference from the extracted one. Therefore, the watermarking part works properly. On the other hand, we also need to evaluate the influence of watermark embedding on hash re-computation $I_{w h}$. We compare the re-computed hash values with and without watermarking, and list the average distances (BERs) in Table 7 . The results show that the influence is quite small. This is expected, because hashing and watermarking are carried out in different wavelet subbands.

Table 8 shows the robustness test results. Among the incidental distortion, except for JPEG 10 and gamma correction 0.5, the average hash distances are all quite small (less than 0.1). They indicate strong resistance to incidental distortion. Regarding malicious distortion, note that besides AWGN 20 dB, JPEG 10, and gamma correction 0.5 , the rest of incidental distortion results smaller hash distances than the distances by tampering two or more blocks. Therefore, the current configuration is able to distinguish incidental and malicious distortion in most cases. If better distinction is required, one may use a larger hash size.

Figure 2 shows the actual distribution of hash distances in the discrimination test. They are derived from 6415200 hash comparisons between different images. The hash values are assumed to be independent of each other. Ideally, the distribution of the normalized Hamming distances is binomial with $p=0.5$ and $N=128$. The empirical distribution has a standard deviation 0.0519 , with a mean of 0.4957. Most hash distances for different images are close to 0.5, which is close to the ideal situation. Since the standard deviation of a binomial distribution is given by $\sigma=\sqrt{p(1-p) / N}$, the distribution of hash distances 
Table 6. Robustness of watermark embedding for $\{n=64, \Delta=120\}$. Original hash values are compared with extracted ones.

\begin{tabular}{l|l|l|l|l|l|l|l}
\hline AWGN & 20 & 25 & 30 & 35 & 40 & 45 & 50 \\
\hline Mean BER & .069 & .000 & 0 & 0 & 0 & 0 & 0 \\
\hline \hline JPEG & 10 & 20 & 30 & 40 & 50 & 60 & 70 \\
\hline Mean BER & .283 & .007 & .007 & .017 & .072 & .000 & .000 \\
\hline \hline Gaussian filt. & 7 & 9 & 11 & 13 & 15 & 17 & 19 \\
\hline Mean BER & 0 & 0 & 0 & 0 & 0 & 0 & 0 \\
\hline \hline Sharpening & 0.1 & 0.2 & 0.3 & 0.4 & 0.5 & 0.6 & 0.7 \\
\hline Mean BER & .021 & .020 & .020 & .019 & .019 & .019 & .019 \\
\hline \hline Gamma corr. & 0.5 & 0.7 & 0.9 & 1.1 & 1.3 & 1.5 & 1.7 \\
\hline Mean BER & .068 & .032 & 0 & 0 & .001 & .005 & .013 \\
\hline \hline Block tampering & 1 & 2 & 3 & 4 & 5 & 6 & 7 \\
\hline Mean BER & 0 & 0 & 0 & .001 & .001 & .002 & .002 \\
\hline
\end{tabular}

Table 7. Influence of watermark embedding on hash re-computation for $\{n=64, \Delta=$ $120\}$. The re-computed hash values with and without watermarking are compared.

\begin{tabular}{l|l|l|l|l|l|l|l}
\hline AWGN & 20 & 25 & 30 & 35 & 40 & 45 & 50 \\
\hline Mean BER & .013 & .008 & .005 & .003 & .001 & .001 & .001 \\
\hline \hline JPEG & 10 & 20 & 30 & 40 & 50 & 60 & 70 \\
\hline Mean BER & .001 & .001 & .002 & .002 & .001 & .002 & .002 \\
\hline \hline Gaussian filt. & 7 & 9 & 11 & 13 & 15 & 17 & 19 \\
\hline Mean BER & .001 & .001 & .001 & .001 & .001 & .001 & .001 \\
\hline \hline Sharpening & 0.1 & 0.2 & 0.3 & 0.4 & 0.5 & 0.6 & 0.7 \\
\hline Mean BER & .001 & .001 & .001 & .001 & .001 & .001 & .001 \\
\hline \hline Gamma corr. & 0.5 & 0.7 & 0.9 & 1.1 & 1.3 & 1.5 & 1.7 \\
\hline Mean BER & .002 & .002 & .001 & .001 & .001 & .001 & .001 \\
\hline \hline Block tampering & 1 & 2 & 3 & 4 & 5 & 6 & 7 \\
\hline Mean BER & .143 & .005 & .004 & .005 & .006 & .005 & .005 \\
\hline
\end{tabular}

corresponds to a binomial process with $N=92$ and $p=0.5$, whose theoretical distribution is plotted by a solid line in Fig. 2. One can see that the theoretical distribution approximately fits the actual data. Therefore, 92 out of $128(72 \%)$ hash bits are independent and unpredictable. The probability that two random image blocks have the same hash value is about $2^{-92}$. If a lower collision rate is required, one could use a larger hash size.

Figure 3 and 4 show the hypothesis test results. A state-of-the-art algorithm [7] is implemented as a baseline for performance comparison. This scheme was shown in [7] to outperform Fridrich's scheme [3]. We configure it to have the same hash size (128 bits) as the proposed scheme, and test it in the same way. One can see efficient trade-offs between the false positive rate and the false negative rate from the figures. Some typical values of the ROC curve are listed in Table 9. Our scheme can achieve a true positive rate above 0.9 while maintains a false 
Table 8. Robustness test for $\{n=64, \Delta=120\}$.

\begin{tabular}{l|l|l|l|l|l|l|l}
\hline AWGN & 20 & 25 & 30 & 35 & 40 & 45 & 50 \\
\hline Mean BER & .082 & .009 & .006 & .003 & .002 & .002 & .001 \\
\hline \hline JPEG & 10 & 20 & 30 & 40 & 50 & 60 & 70 \\
\hline Mean BER & .290 & .013 & .013 & .022 & .073 & .003 & .003 \\
\hline \hline Gaussian filt. & 7 & 9 & 11 & 13 & 15 & 17 & 19 \\
\hline Mean BER & .003 & .003 & .003 & .003 & .003 & .003 & .003 \\
\hline \hline Sharpening & 0.1 & 0.2 & 0.3 & 0.4 & 0.5 & 0.6 & 0.7 \\
\hline Mean BER & .045 & .045 & .044 & .044 & .043 & .044 & .043 \\
\hline \hline Gamma corr. & 0.5 & 0.7 & 0.9 & 1.1 & 1.3 & 1.5 & 1.7 \\
\hline Mean BER & .119 & .062 & .012 & .011 & .030 & .050 & .074 \\
\hline \hline Block tampering & 1 & 2 & 3 & 4 & 5 & 6 & 7 \\
\hline Mean BER & .047 & .081 & .101 & .166 & .181 & .192 & .196 \\
\hline
\end{tabular}

Table 9. Typical values of the ROC curves for the proposed scheme $\{n=64, \Delta=120\}$ and the baseline scheme [7].

\begin{tabular}{l|ll}
\hline \multirow{2}{*}{$P_{f}$} & \multicolumn{2}{|c}{$P_{d}$} \\
\cline { 2 - 3 } & proposed & baseline \\
\hline $10^{-1}$ & 0.996 & 0.977 \\
$10^{-2}$ & 0.990 & 0.945 \\
$10^{-3}$ & 0.982 & 0.814 \\
$10^{-4}$ & 0.974 & 0.366 \\
$10^{-5}$ & 0.973 & $\ldots$ \\
$10^{-6}$ & 0.972 & $\ldots$ \\
$10^{-7}$ & 0.912 & $\ldots$ \\
\hline
\end{tabular}

positive rate below $10^{-7}$. These results prove that the proposed system works properly and significantly outperforms the baseline algorithm.

\section{Conclusion}

Robust hashing is a promising solution to the multimedia content authentication problem. It can be used together with digital watermarking techniques. Instead of being transmitted separately, hash values can be embedded in the corresponding multimedia data. The advantage is that no extra communication is required the original hash value is always available and synchronized. This makes robust hashing a more versatile solution. However, on the other hand, image quality can be degraded due to watermark embedding.

There is interesting interaction between hashing and watermarking. We investigate this issue by proposing a content authentication system. The hash algorithm and the watermarking algorithm are designed to have minimal interference. This is achieved by doing hashing and watermarking in different wavelet subbands. We define the authentication performance as the receiver operating 


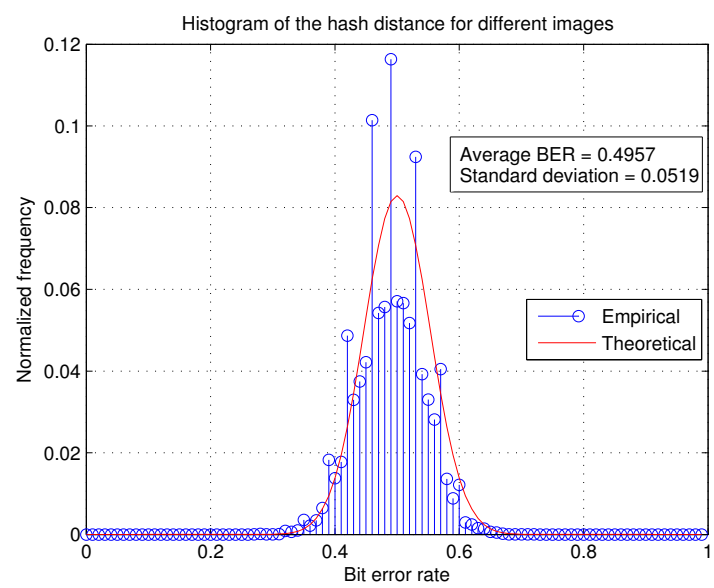

Fig. 2. BER Histogram for different images for $\{n=64, \Delta=120\}$.

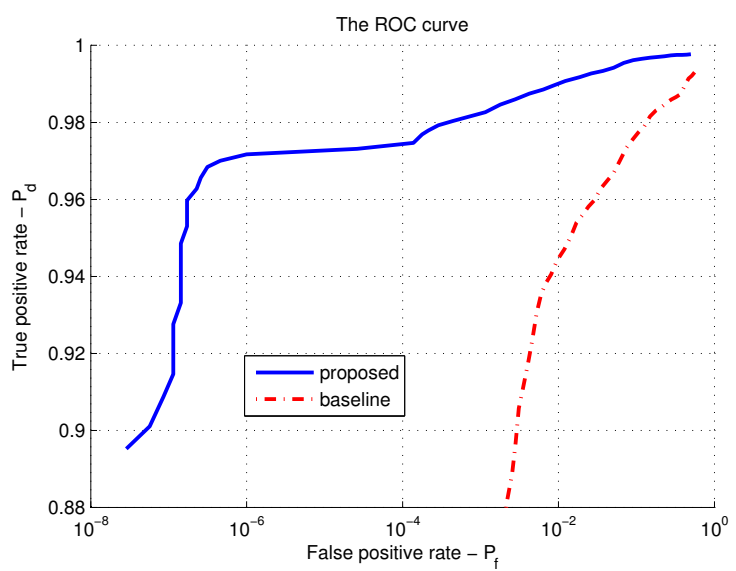

Fig. 3. The ROC curves for the proposed scheme $\{n=64, \Delta=120\}$ and the baseline scheme [7]. 


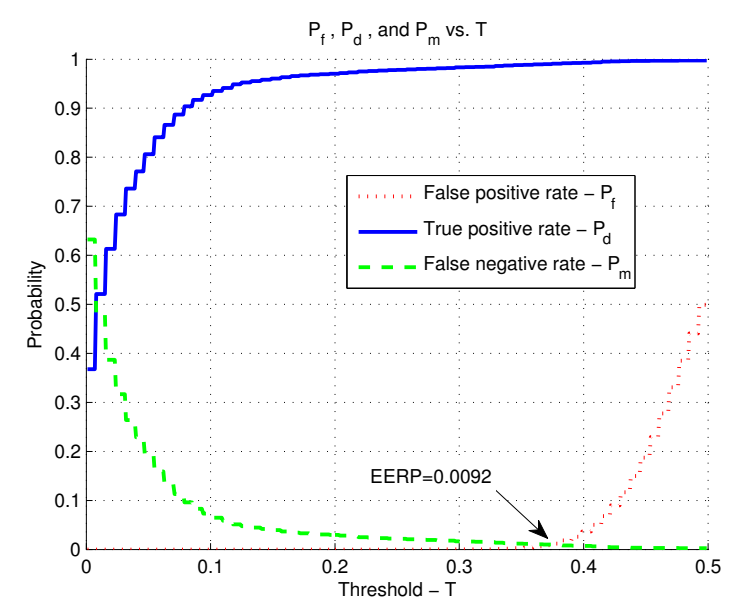

Fig. 4. Hypothesis test results for $\{n=64, \Delta=120\}$.

characteristics of the hash algorithm. Through extensive experiments we show that the parameters of the watermarking algorithm have significant influence to the authentication performance. There is a trade-off between the authentication performance and the perceptual quality. In practice, we can fix one of them and try to get the best performance for the other.

The proposed algorithm is able to distinguish incidental and malicious distortion. It significantly outperforms a state-of-the-art algorithm by achieving a true positive rate above 0.9 while maintaining a false positive rate below $10^{-7}$. Our work gives useful insights into the research and practice in this field.

\section{References}

1. Schneier, B.: Applied Cryptography: Protocols, Algorithms, and Source Code in C. 2nd edn. John Wiley \& Sons (1996)

2. Swaminathan, A., Mao, Y., Wu, M.: Robust and secure image hashing. IEEE Transactions on Information Forensics and Security 1(2) (June 2006) 215-230

3. Fridrich, J.: Robust bit extraction from images. In: Proc. of IEEE International Conference on Multimedia Computing and Systems. Volume 2. (1999) 536-540

4. Fridrich, J., Goljan, M.: Robust hash functions for digital watermarking. In: Proc. of International Conference on Information Technology: Coding and Computing. (2000) 178-183

5. Chen, B., Wornell, G.: Quantization index modulation: a class of provably good methods for digital watermarking and information embedding. IEEE Transactions on Information Theory 47(4) (May 2001) 1423-1443

6. Wang, Z., Bovik, A., Sheikh, H., Simoncelli, E.: Image quality assessment: From error visibility to structural similarity. IEEE Transactions on Image Processing 13(4) (April 2004) 600-612

7. Khelifi, F., Jiang, J.: Perceptual image hashing based on virtual watermark detection. IEEE Transactions on Image Processing 19(4) (April 2010) 981-994 